\title{
Modeling Chemical Detection Sensitivities of Active and Passive Remote Sensing Systems
}

\author{
E. T. Scharlemann
}

This article was submitted to the $48^{\text {th }}$ Annual Meeting of the SPIE, San Diego, CA, August 3-8, 2003

U.S. Department of Energy July 28, 2003

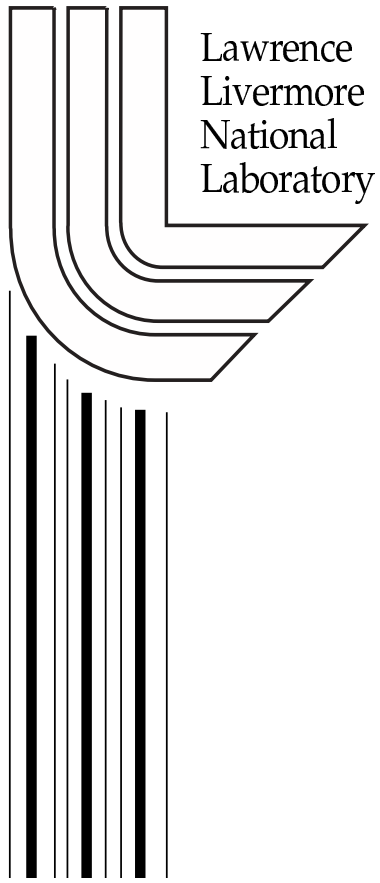




\section{DISCLAIMER}

This document was prepared as an account of work sponsored by an agency of the United States Government. Neither the United States Government nor the University of California nor any of their employees, makes any warranty, express or implied, or assumes any legal liability or responsibility for the accuracy, completeness, or usefulness of any information, apparatus, product, or process disclosed, or represents that its use would not infringe privately owned rights. Reference herein to any specific commercial product, process, or service by trade name, trademark, manufacturer, or otherwise, does not necessarily constitute or imply its endorsement, recommendation, or favoring by the United States Government or the University of California. The views and opinions of authors expressed herein do not necessarily state or reflect those of the United States Government or the University of California, and shall not be used for advertising or product endorsement purposes.

This is a preprint of a paper intended for publication in a journal or proceedings. Since changes may be made before publication, this preprint is made available with the understanding that it will not be cited or reproduced without the permission of the author.

Available electronically at http://www.doc.gov/bridge

Available for a processing fee to U.S. Department of Energy

And its contractors in paper from

U.S. Department of Energy

Office of Scientific and Technical Information

P.O. Box 62

Oak Ridge, TN 37831-0062

Telephone: (865) 576-8401

Facsimile: (865) 576-5728

E-mail: reports@adonis.osti.gov

Available for the sale to the public from

U.S. Department of Commerce

National Technical Information Service

5285 Port Royal Road

Springfield, VA 22161

Telephone: (800) 553-6847

Facsimile: (703) 605-6900

E-mail: orders@ntis.fedworld.gov

Online ordering: http://www.ntis.gov/ordering.htm

OR

Lawrence Livermore National Laboratory

Technical Information Department's Digital Library

http://www.llnl.gov/tid/Library.html 


\title{
Modeling chemical detection sensitivities of active and passive remote sensing systems*
}

\author{
E. T. Scharlemann \\ Lawrence Livermore National Laboratory, L-183 \\ Livermore, California 94550
}

\begin{abstract}
During nearly a decade of remote sensing programs under the auspices of the U. S. Department of Energy (DOE), LLNL

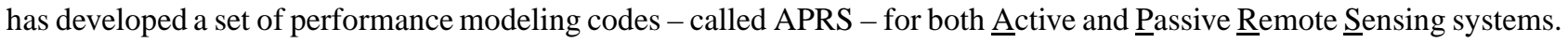
These codes emphasize chemical detection sensitivity in the form of minimum detectable quantities with and without background spectral clutter and in the possible presence of other interfering chemicals. The codes have been benchmarked against data acquired in both active and passive remote sensing programs at LLNL and Los Alamos National Laboratory (LANL). The codes include, as an integral part of the performance modeling, many of the data analysis techniques developed in the DOE's active and passive remote sensing programs (e.g., "band normalization" for an active system, principal component analysis for a passive system).
\end{abstract}

Keywords: DIAL, remote sensing, modeling

\section{INTRODUCTION}

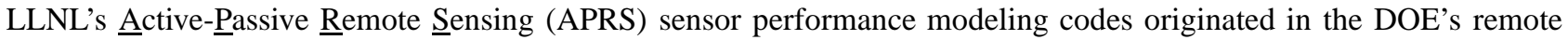
sensing trade studies lasting from November of 1997 through August of 2001. In the study, DOE's MWIR and LWIR active and passive remote sensing programs were initially found to be nearly impossible to compare; each program made performance calculations based on its own set of assumptions, usually orthogonal to every other program's assumptions. The APRS codes were constructed specifically to ensure that all the performance modeling calculations were using consistent assumptions and treating such effects as overlap between a chemical plume and the light source in the same way. The codes were extensively benchmarked during the trade study effort with CALIOPE (active) data from both LANL (LWIR) and LLNL (MWIR), and passive data from LLNL and Aerospace Corp. (both LWIR).

The intent of the codes is to provide a capability for rapid examination of potential system parameter trades; the codes are not detailed engineering design codes. The codes are primarily aimed at calculating chemical detection sensitivities for sensors on moving platforms using the atmospheric windows from 8-12 $\mu \mathrm{m}$ and 3-5 $\mu \mathrm{m}$. In this paper, we describe the equations used in the models and some of the built-in analysis algorithms.

\section{PASSIVE SENSORS - DISPERSIVE, FOURIER-TRANSFORM AND FABRY-PEROT SPECTROMETERS}

The sources of signal photons - those susceptible to absorption by a gaseous plume - are thermal greybody emission from the ground and reflected sunlight. The emissivity $\epsilon$ and reflectivity $\rho=1-\epsilon$ of the ground are assumed to be spectrally constant. The instrument is characterized by an étendue $E\left(\mathrm{~cm}^{2}-\mathrm{sr}\right)$, an optical throughput $\eta_{\text {opt }}$, and a detector quantum efficiency $\eta_{Q}$, from which a signal photoelectron count is derived. Quantitatively, with blackbody spectral radiance from the ground

$$
\mathcal{B}_{G}\left(\nu, T_{G}\right)=1.1911 \times 10^{-12} \frac{\nu^{3}}{e^{1.4384 \nu / T_{G}}-1} \quad \mathrm{~W} / \mathrm{cm}^{2}-\mathrm{sr}-\mathrm{cm}^{-1},
$$

*To be published in Proc. SPIE 5154 (2003). 
(where $\nu$ is in $\mathrm{cm}^{-1}$ and $T_{G}$ is the temperature of the ground in $\mathrm{K}$ ), the signal photoelectron count from thermal emission from the ground (the signal that is absorbed by a plume) into spectral channel $j$ is

$$
n_{G j}=\epsilon \mathcal{B}_{G}\left(\nu_{j}, T_{G}\right) T_{a t m}\left(\nu_{j}\right) \dot{E} \eta_{o p t} \eta_{Q} T_{i n t} \delta \nu_{j} \frac{\lambda_{j}}{h c} \quad \text { photoelectrons. }
$$

Here, $\delta \nu_{j}$ is the width of the spectral channel (in $\mathrm{cm}^{-1}$ ), $T_{a t m}\left(\nu_{j}\right)$ is the atmospheric transmission (from FASCODE ${ }^{1,2}$ and HITRAN $2000^{3,4}$ ) averaged over $\delta \nu_{j}$, and $T_{i n t}$ is the integration time. For a Fourier-transform spectrometer (FTS), $\delta \nu_{j}$ is independent of $\nu_{j}$. For a dispersive instrument, $\delta \nu_{j}$ depends on the design of the grating. Three options are provided by APRS: $\delta \nu_{j}$ linearly proportional to $\nu_{j}, \delta \lambda_{j}$ constant, or $\nu_{j}$ and $\delta \nu_{j}$ specified in an external file. The étendue $E$ is the product of the area of the single-pixel IFOV on the ground and the solid angle of the receiver telescope as seen from the ground; it determines the total amount of light that reaches each pixel. The optical throughput $\eta_{\text {opt }}$ would include $~ 50 \%$ losses at an FTS beam-splitter.

For daytime MWIR modeling, sunlight provides an additional source of signal photons. Insolation $\left(\mathcal{S}(\nu)\right.$ in W/cm $\left.-\mathrm{cm}^{-1}\right)$ at the top of the atmosphere is taken from LOWTRAN7, and converted to spectral radiance from a Lambertian surface by multiplying by $\rho \cos \psi / \pi$, where $\psi$ is the zenith angle of the sun as seen from the ground. Atmospheric transmission is included for both traverses of the atmosphere by the sunlight, $T_{a t m, d o w n}\left(\nu_{j}\right)$ and $T_{a t m, u p}\left(\nu_{j}\right)$; these are not identical because the downward and upward paths need have neither the same lengths nor zenith angles.

$$
n_{S j}=\frac{\rho \cos \psi}{\pi} \mathcal{S}\left(\nu_{j}\right) T_{a t m, \text { down }}\left(\nu_{j}\right) T_{a t m, \text { up }}\left(\nu_{j}\right) \dot{E} \eta_{\text {opt }} \eta_{Q} T_{i n t} \delta \nu_{j} \frac{\lambda_{j}}{h c} \quad \text { photoelectrons. }
$$

The noise terms included in the model are

- shot noise from instrumental background (thermal emission from lenses, walls, and mirrors, characterized simply by an effective temperature and emissivity), atmospheric radiance (again from FASCODE), and the signal itself,

- shot noise from the detector dark current $\left(\mathrm{e}^{-/ \mathrm{s}}\right)$,

- read-out noise $\left(N_{\text {read }}, \mathrm{rms} \mathrm{e}^{-} / \mathrm{read}\right)$.

The detector read time is limited by the charge capacity (well depth) of the pixels and the fill rate from the dark current and the photoelectron generation terms. The maximum fill rate over all spectral channel is used to set the read time in a dispersive instrument. Atmospheric radiance enters into the noise but not the signal because most of the atmospheric radiance arises from the path between plume and instrument, and so will not be absorbed by the plume.

The instrumental background is

$$
n_{I j}=\epsilon_{I} \mathcal{B}_{I}\left(\nu_{j}, T_{I}\right) \dot{E} \eta_{o p t} \eta_{Q} T_{i n t} \delta \nu_{j} \frac{\lambda_{j}}{h c} \quad \text { photoelectrons, }
$$

where $\epsilon_{I}$ is an effective instrumental emissivity, and $\mathcal{B}_{I}\left(\nu_{j}\right)$ is the blackbody spectral radiance at the effective instrumental background temperature $T_{I}$. The use of $\delta \nu_{j}$ in Eq. 4 assumes that the dominant instrumental background arises from the fore-optics (before the slit preceding the dispersive element) in a dispersive system; else the full cold-filter bandwidth $\Delta \nu$ needs to be used.

Atmospheric radiance contributes

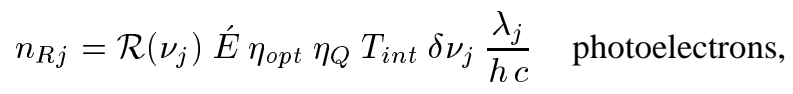

where $\mathcal{R}\left(\nu_{j}\right)$ is the upwelling spectral radiance of the atmosphere in $\mathrm{W} / \mathrm{cm}^{2}-\mathrm{sr}-\mathrm{cm}^{-1}$ from FASCODE.

For the FTS, the shot noise term includes shot noise from the entire spectral passband, and an additional reduction in signal-to-noise ratio (SNR) of $\sqrt{2}$ is included because of the way noise propagates through the cosine transform used to convert interferograms to spectra. ${ }^{5}$ The read time for the FTS detector is set using the photoelectron generation rate of the entire spectral passband. 
For both dispersive or FTS sensors, the FPA requires calibration to correct for FPA non-uniformities and drifts. The calibration cycle will have its own noise, which propagates into the noise on each spectral channel. The resulting increase in noise will depend on many details; for the modeling described here, the increase is taken to be negligible, which presupposes that noise in the calibration cycle is reduced well below the noise during a scene measurement.

The raw photon signal-to-noise ratio (SNR) for an FTS is then

$$
S N R_{j}^{F T S, \text { raw }}=\frac{n_{G j}}{\sqrt{2}\left(N_{T}+\frac{T_{\text {int }}}{t_{\text {read }}} N_{\text {read }}^{2}+N_{\text {dark }}\right)^{1 / 2}},
$$

where

$$
N_{T}=\sum_{j}\left(n_{G j}+n_{R j}+n_{S j}+n_{I j}\right)
$$

and, for a focal-plane array well depth of $W_{e}$,

$$
t_{\text {read }}=\frac{0.5 W_{e}}{\left(N_{T}+N_{\text {dark }}\right) / T_{\text {int }}} ;
$$

that is, the FPA is read out when the wells are half full.

For a dispersive imager, the raw photon signal-to-noise ratio (SNR) is

$$
S N R_{j}^{\text {Disp,raw }}=\frac{n_{G j}}{\left(n_{T j}+\frac{t_{\text {int }}}{t_{\text {read }}} N_{\text {read }}^{2}+N_{\text {dark }}+N_{\text {cal }}^{2}\right)^{1 / 2}}
$$

(no $\sqrt{2}$ ), where

$$
n_{T j}=n_{G j}+n_{R j}+n_{S j}+n_{I j}
$$

and

$$
t_{\text {read }}=\frac{0.5 W_{e}}{\left[\operatorname{Ma}\left(n_{T j}\right)+N_{\text {dark }}\right] / t_{\text {int }}} ;
$$

that is, the FPA is read out when the most rapidly filled well is half full. The integration time for a single across-track line of pixels for the imager is $t_{i n t}=T_{\text {int }} / N_{\text {image pixels }}$, where $N_{\text {image pixels }}$ is the number of along-track image pixels built up in a pushbroom scan of the full image time $T_{i n t}$. Each of the terms $n_{G j}, n_{R j}, n_{S j}$, and $n_{I j}$ in Eqs. 9-11 is determined using $t_{\text {int }}$ instead of $T_{\text {int }}$.

An additional error term, $N_{c a l}$, representing pixel-to-pixel calibration error of the FPA, has been introduced here. It is inspired by the semi-quantitative notion that the best one can do in achieving pixel-to-pixel uniformity in a featureless blackbody spectrum is about a part in $10^{4}$; hence $N_{c a l} \approx 10^{-4} \times\left(n_{T j}+N_{d a r k}\right)$. This "noise" source has a significant impact on a high-SNR non-imaging or partially-imaging dispersive sensor, and so could use a much better model than this rather simple attempt.

For the non-imaging (or partially-imaging, with limited spatial information) dispersive spectrometer, $t_{i n t}=T_{\text {int }}$ because the full $T_{i n t}$ is assumed to be spent staring at a single ground position.

An imaging Fabry-Perot spectrometer is modeled in the same way as the dispersive system, with two minor modifications. First, $t_{i n t}$ in Eq. 9 is set by the time spent on each spectral channel rather than on a row of spatial pixels. Second, there is generally some duty factor $D_{f}$ less than unity for the Fabry-Perot spectrometer, set by the settling time of a piezoelectrically-driven filter for example. Hence, the same equation (Eq. 9) for SNR can be used for the Fabry-Perot, but with $t_{\text {int }}=D_{f} \times T_{\text {int }} / N_{\text {spectral channels. }}$.

Spectral "dilution" of the plume signature is included by integrating the product of high-resolution chemical and atmospheric spectra over the spectral bins of the instrument. These bins are assumed to be square-edged, but the use of a more 
accurate spectral profile has been tried and makes little difference. For chemical $m$, the "diluted" absorption in spectral bin $j$ is

$$
\tau_{j}^{m}=\frac{\int_{\delta \nu_{j}} T_{a t m}(\nu) \tau^{m}(\nu) d \nu}{\int_{\delta \nu_{j}} T_{a t m}(\nu) d \nu} .
$$

Chemical spectra are taken from the standard databases, primarily the Hanst Library from Infrared Analysis, Inc., ${ }^{6}$ and the Pacific Northwest National Laboratory library. ${ }^{7}$

The radiance contrast between plume and ground is characterized by a brightness temperature differential $\delta T_{B}$, assumed to be a spatial and temporal average over the spot on the ground seen by a pixel and the integration time. Since for small concentrations and $\delta T_{B}$ near detection threshold, the absorption is linear in the chemical concentration and $\delta T_{B}$, the use of an average $\delta T_{B}$ is legitimate. At higher absorptions or greater temperature differentials, non-linearities obviously would have to be included, but these non-linearities are not important for the comparisons for which the models were developed. The specified $\delta T_{B}$ includes both a physical ground-plume difference and a ground emissivity less than unity; the ground emissivity $\epsilon$ mentioned above does not also enter into the radiance contrast calculation. The radiance contrast $C$ is

$$
C=\frac{h c}{\lambda k T} \frac{\delta T_{B}}{T_{B}},
$$

and just comes from the derivative of the expression for blackbody spectral radiance.

The "raw photon" SNR, radiance contrast, and a number $N_{\text {pixels }}$ of pixels over which one can successfully average the spectral information to reduce the noise, combine to yield an SNR for each spectral channel:

$$
S N R_{j}=C \times \sqrt{N_{\text {pixels }}} \times S N R_{j}^{\text {raw }}
$$

in the LWIR, or

$$
S N R_{j}=\sqrt{N_{\text {pixels }}} \frac{C n_{G j}+n_{S j}}{\text { noise terms }}
$$

in the daytime MWIR when reflected sunlight is important; the radiance contrast does not affect the solar term (although since $C$ can be negative, it is perfectly possible for $S N R_{j}$ to vanish in some spectral channels).

The noise is assumed to be uncorrelated among the spectral channels. Standard weighted-least-squares techniques are then used to evaluate a 1- $\sigma$ uncertainty in the accuracy with which a chemical's concentration-pathlength product (CL) can be determined; this 1- $\sigma$ uncertainty is the NECL, or noise-equivalent concentration-pathlength product. The mathematical procedure is straight-forward. An $N_{\text {bins }} \times M_{\text {chemicals }}$ absorption matrix $\mathbf{K}$ is constructed from the $M_{\text {chemicals }}$ chemicals being looked for:

$$
K_{j m}=\tau_{j}^{m}
$$

note that atmospheric transmission is included in the $\tau_{j}^{m}$ as indicated in Eq. 12. The $S N R_{j}$ appear in an $N_{\text {bins }} \times N_{\text {bins }}$ noise-covariance matrix $\Lambda$ :

$$
\begin{aligned}
& \boldsymbol{\Lambda}=\operatorname{diag}\left(\sigma_{j}^{2}\right) \quad \text { with } \\
& \sigma_{j}^{2}=\frac{1}{S N R_{j}^{2}} .
\end{aligned}
$$

The absorption and noise covariance matrices combine to give an $M_{\text {chemicals }} \times M_{\text {chemicals }}$ CL covariance matrix $\boldsymbol{\Lambda}_{C L}$ :

$$
\mathbf{\Lambda}_{C L}=\left(\mathbf{K}^{T} \mathbf{\Lambda}^{-1} \mathbf{K}\right)^{-1}
$$

and the diagonal elements of $\boldsymbol{\Lambda}_{C L}$ are the squares of the NECLs without accounting for spatial dilution; i.e., the fact that the plume is generally smaller than the average target spot size $D_{\text {spot }}$ on the ground. With this matrix technique, these NECLs are calculated for all chemicals in specified suites in combination, not individually. 
The overlap between plume and diffraction-limited pixel spot on the ground (not the IFOV: generally the spot on the ground covered by a pixel's Airy disk is larger than the IFOV) is accounted for by 1) convolving a Gaussian jitter term (1-axis, $1-\sigma$ ) with the Airy disk and turbulent image blur to produce a quasi-Gaussian ground pattern, and 2) increasing the NECL by the term appropriate for a cylindrical plume threading the quasi-Gaussian ground spot. Specifically, the NECLs are increased by $\frac{3 \pi}{8 \sqrt{2}} \frac{D_{\text {spot }}}{D_{\text {plume }}}$, with $D_{\text {spot }}$ determined from the aperture size, the jitter, and the turbulence as follows.

The turbulent image blurring is characterized by Fried's coherence length $r_{0}$, given in SI units $\left(r_{0}\right.$ in m) by ${ }^{8}$

$$
r_{0}=2.1\left[1.46 k^{2} \int_{0}^{L} C_{n}^{2}(s)\left(\frac{s}{L}\right)^{5 / 3} d s\right]^{-3 / 5}
$$

for a spherical wave propagating from $s=0$ to $L \mathrm{~m}$, appropriate to imaging the ground ( $k$ is $\frac{2 \pi}{\lambda}$ in $\mathrm{m}^{-1}$, and $C_{n}^{2}$ is the refractive-index structure constant in $\mathrm{m}^{-2 / 3}$; a Hufnagel-Valley model with boundary layer ${ }^{9}$ is used for the dependence of $C_{n}^{2}$ on altitude). The diffraction blur is then the Airy disk for an aperture with diameter $D_{\text {ef } f}$ given by

$$
D_{\text {eff }}=\frac{1}{1 / D_{r}+1 / r_{0}} .
$$

The effective quasi-Gaussian spot size is

$$
\bar{D}_{\text {spot }}=1.8 \frac{\lambda L}{D_{\text {eff }}} .
$$

This is convolved with the pointing jitter $\left(\sigma_{P J}\right)$ to give a final spot diameter of

$$
D_{\text {spot }}=\sqrt{\bar{D}_{\text {spot }}^{2}+16 \sigma_{P J}^{2}}
$$

Finally, the NECLs are optionally increased by an additional factor of $\sqrt{2}$ to account for an explicit or implicit ratioing or differencing of on-plume pixels to off-plume pixels. This ratioing or differencing is necessary - if for no other reason to normalize out atmospheric absorption. If the more extended scene analysis described below in Section 4 is used, this factor is not included, with the rationale that much more information is available about off-plume pixels, hence off-plume noise is reduced well below on-plume noise.

Not included in the passive sensor models are:

- the effect of jitter combined with scene contrast on FTS interferograms,

- uncertainties in radiance contrast,

- inaccuracies in the atmospheric model used to obtain $\tau_{j}^{m}$,

- effects of line-of-sight variation during data collection,

- image keystoning,

- optical depth effects (non-linear absorption),

- down-welling atmospheric radiance reflected from the ground,

- multiple scattering,

- A/D digitization errors,

- pixel cross-talk, and

- optical aberrations or vignetting.

Reflected down-welling radiation is unimportant except in the very scene-dependent case of a highly reflective background pixel.

\section{ACTIVE SENSORS}

A similar procedure is followed for the active systems, with differences appropriate to the nature of the photon source. Individual laser pulses are characterized by a frequency, a pulse energy $E_{p u l s e}$, and a mirror fill-factor that specifies the 
amount of energy truncated by the transmitting mirror and influences the details of the target spot shape (which enters later in the speckle noise calculation). In addition to the mirror truncation factor, the system has an overall hardware optical throughput and a detector quantum efficiency. Atmospheric transmission (round trip) is obtained from FASCODE, with no integration over the laser linewidth - the lines are assumed to be narrow enough and located in wide enough transmission windows to permit this. One of the inputs to the model is a list of laser line frequencies; this input also sets the number of laser lines.

The ground is treated as a Lambertian diffuse reflector with a reflectivity $\rho$, and a $\cos \theta$ factor is included for reduction in return photons for off-nadir viewing angles.

The result of these factors is a return signal photoelectron count for each laser pulse on line $l$ :

$$
n_{l}=E_{\text {pulse }} \frac{\lambda_{l}}{h c} \frac{\rho \cos \theta}{\pi}\left(\frac{\pi}{4} \frac{D_{R}^{2}}{L^{2}}\right) T_{\text {trunc }} T_{\text {atm }}^{2}\left(\nu_{l}\right) \eta_{\text {opt }} \eta_{Q},
$$

where $T_{\text {trunc }}$ is the loss of laser pulse energy by truncation at the transmitting mirror. For a Gaussian beam with $1 / e^{2}$ radius of $w$ and a transmitting mirror of radius $b=D_{T} / 2$, the truncation factor is

$$
T_{\text {trunc }}=1-e^{-2 b^{2} / w^{2}}
$$

The $D_{R}^{2}$ in Eq. 24 needs to be replaced by $D_{R}^{2}-D_{T}^{2}$ for a coaxial transmitter/receiver configuration: $D_{R}$ is the receiver mirror diameter and $D_{T}$ is the transmitter mirror diameter. Eq. 24 implicitly assumes that the detector FOV is large enough to encompass the entire laser spot on the ground.

Diffraction of the truncated beam in propagating to the ground is characterized by using an effective $1 / e^{2}$ radius $w_{\text {eff }}$ at the transmitting mirror given by

$$
w_{e f f}=w\left[\frac{1-\left(1+\frac{b^{2}}{w^{2}}\right) e^{-b^{2} / w^{2}}}{1-e^{-b^{2} / w^{2}}}\right]^{1 / 2} .
$$

This form is obtained simply by looking at the first two terms in the Taylor series expansion of the exact far-field expression for a truncated Gaussian beam, but more careful numerical analysis has shown that results using Eq. 26 accurately characterize both speckle noise and plume dilution in the resulting target spot.

Turbulent beam spreading is included through the transverse coherence length $\rho_{0}$ given by

$$
\rho_{0}=\left[1.46 k^{2} \int_{0}^{L} C_{n}^{2}(s)\left(1-\frac{s}{L}\right)^{5 / 3} d s\right]^{-3 / 5}
$$

for a beam propagating from $s=0$ to $L$. The final target spot size on the ground, with both diffraction and turbulent beam spreading, is

$$
\bar{D}_{\text {target }}^{2}=\frac{16 L^{2}}{k^{2} w_{\text {eff }}^{2}}+4 w_{\text {eff }}^{2}\left(1-\frac{L}{f}\right)^{2}+\frac{32 L^{2}}{k^{2} \rho_{0}^{2}} .
$$

with $f$ the wavefront radius of curvature at the transmitter mirror. For atmospheric path lengths $L$ such that $L>>D^{2}$, (Fante's "Case 4" 10 ) this expression is evidently appropriate for both short-term and long-term beam spread. Eq. 28 is slightly different from the expression used in LANL's SONDIAL model ${ }^{11}$ but is, in the author's opinion, slightly more correct. The differences in the final results are minimal.

Noise is included through four terms:

- speckle,

- combined detector and pre-amp noise, specified as an r.m.s. electron noise count by the detector builders,

- return signal shot noise, 
- shot noise in the filtered and time-gated background, solar plus thermal.

The speckle noise model is the result of a detailed analysis of the speckle SNR from a quasi-Gaussian ground spot in a circular aperture - the receiver telescope (note that speckle noise can be calculated either at the receiver aperture or on the detector and that if the system is designed properly, both answers will be identical). The speckle correlation length (or speckle-cell diameter) is

$$
D_{\text {corr }}=\frac{8 L}{k \bar{D}_{\text {target }}} .
$$

The number of speckle cells in the receiver aperture is

$$
\begin{aligned}
M_{\text {speckle }} & =1+1.088 \frac{D_{R}^{2}-D_{T}^{2}}{D_{\text {corr }}^{2}} \quad \text { coaxial } \\
& =1+1.088 \frac{D_{R}^{2}}{D_{\text {corr }}^{2}} \quad \text { non-coaxial. }
\end{aligned}
$$

The factor of 1.088 comes from a fit to numerical evaluation of Eq. 2.114 of Ref. ${ }^{12}$ for a circular aperture and Gaussian spot.

The combination of a non-zero laser linewidth and surface roughness or tilt can reduce speckle noise by increasing $M_{\text {speckle }}$. Since surface roughness is so highly scenario dependent, only surface tilt effects are treated (surface roughness can be considered to provide an equivalent tilt). A diffusely-scattering surface with normal direction tilted at angle $\theta$ from the laser propagation direction produces a frequency decorrelation of the speckle (simply by a spatial shift of the speckle pattern across the receiver aperture) on a scale of

$$
\delta \nu_{c}=\frac{\nu D_{R}}{2 L \tan \theta}
$$

Then $M_{\text {speckle }}$ is increased by a factor $\sqrt{1+\Delta \nu_{L}^{2} / \delta \nu_{c}^{2}}$, where $\Delta \nu_{L}$ is the laser linewidth (which might be $\sim 1 \mathrm{GHz}$, still small compared to atmospheric absorption features or pressure-broadened gas absorption features).

The single-pulse SNR on line $l$ is the inverse of the normalized pulse-return variance $\sigma_{l}^{2}$, given by

$$
\sigma_{l}^{2}=(1+\varepsilon)\left[\frac{n_{e}^{2}}{n_{l}^{2}}+\frac{1}{n_{l}}\right]+\frac{1}{M_{\text {speckle }, l}},
$$

where $n_{e}$ is the combined detector, pre-amplifier, and background thermal/instrumental, etc. noise, in r.m.s. electrons per pulse. The model has the option of separating the detector/pre-amp noise from the background noise, but since calculating the latter requires specifying a detector time gate and cold filter width, it is far more convenient to lump the detector noise terms together into the single quantity $n_{e}$. The factor of $(1+\varepsilon)$ in Eq. 33 arises from the need to normalize the return signal to the fluctuating outgoing laser pulse energy, by ratioing the return signal to a reference signal which will have some detector/pre-amp/background noise but no speckle noise. Because the reference signal can be made much larger than the return signal, its detector/pre-amp/background noise should be (fractionally) much less, so $\varepsilon$ is usually taken to be zero.

Pulse averaging is assumed to increase the SNR for each laser line by $\sqrt{N_{\text {pulses }}}, N_{\text {pulses }}=P R F \times T_{\text {int }}$, as long as the single-line pulse repetition frequency $P R F$ is below the maximum, $P R F_{\max }$, set by requirements for independent speckle patterns on successive pulses at each wavelength:

$$
P R F_{\text {max }}=\frac{V_{\text {platform }}}{D_{R} / 2}
$$

for platform speed $V_{\text {platform }}$. Above that PRF, the speckle noise no longer averages but the detector/amplifier/background noise contributions continue to. 
Usually, the model automatically chooses the optimum trade-off between laser PRF and pulse energy, subject to a specified average laser power and platform velocity. Writing

$$
n_{l}=\alpha E_{\text {pulse }}=\frac{\alpha P_{\text {avg }}}{N_{\text {lines }} P R F}
$$

for a laser average power of $P_{\text {avg }}, N_{\text {lines }}$ laser lines, the optimal $P R F$ is

$$
P R F_{\text {optimal }}=\frac{\alpha P_{\text {avg }}}{\sqrt{(1+\varepsilon) M_{\text {speckle }}} n_{e} N_{\text {lines }}}
$$

for fixed averaging time ( $\alpha$ is the proportionality between $n_{l}$ and $E_{\text {pulse }}$ in Eq. 24). In those (not uncommon) cases for which $P R F_{\text {optimal }}$ is greater than $P R F_{\max }$, the model uses $P R F_{\max }$. Since $\alpha$ depends on atmospheric transmission factors that vary from line to line, an average over all lines is used to determine $P R F_{\text {optimal }}$.

NECLs are calculated as for the passive systems, using an SNR calculated separately for each laser line. ${ }^{13}$ The spatial overlap between plume and target spot is treated almost the same as for the passive systems, with jitter included in the average target spot size:

$$
D_{\text {spot }}=\sqrt{\bar{D}_{\text {target }}^{2}+16 \sigma_{P J}^{2}}
$$

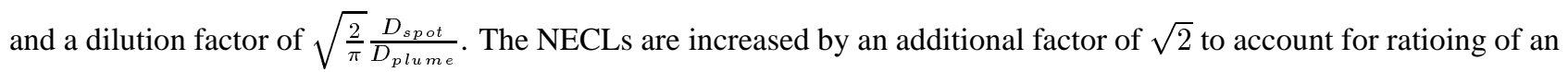
on-plume spot to an off-plume spot, again to normalize out atmospheric absorption.

The multi-line DIAL analysis techniques presented by Warren ${ }^{13}$ involve working with ratios of pairs of return powers, rather then the return powers themselves. This effectively normalizes out the average surface reflectivity of the ground and average atmospheric transmission. The "band-normalization" mentioned in the abstract refers to normalizing out these two factors differently in different regions of the spectrum; $e . g$., in the four separate bands of a $\mathrm{CO}_{2}$ laser (the $\mathrm{P}$ and $\mathrm{R}$ branches with two bands each). Band normalization can reduce sensitivity to the spectral clutter in the background (Bernard Foy [LANL], private communication). The ability to simulate band normalization is built into APRS.

Not included are:

- laser frequency fluctuations and drifts; the laser is assumed to be properly stabilized,

- laser mode structure (which will - to first order - only change the average spot size on the ground),

- turbulent beam breakup on the path to the ground, and its effect on speckle (J. R. Morris, unpublished work),

- return path turbulence (J. R. Morris, unpublished work),

- "albedo noise", an additional pulse-to-pulse noise term that arises from the beam jittering over a surface of spatially non-uniform reflectivity, ${ }^{11}$

- A/D digitization errors,

- optical depth effects,

- misalignments of the optics.

\section{CLUTTER MODELING}

The codes include a simple model for the effects on chemical detectability of background clutter - the spatially variable spectral dependence of scene emissivity, temperature, and reflectivity. The model is based on a database of material reflectivities with specific assumptions about the distribution of those materials across a generic scene. Although simple, the clutter model adequately reproduces the effects of clutter on LLNL's measured active and passive chemical detection sensitivities.

\subsection{Hyperspectral image data analysis}

Each pixel in a hyperspectral image potentially includes radiation from many different materials with spectrally different emissivities. Even in the absence of absorbing gases, the spectral variations introduced by the material emissivities can 
appear to be the result of chemical absorptions. Adjacent pixels are likely to have different sets of materials, limiting the possibilities for removing background spectral features by on- vs off-plume differencing.

Additionally, the backgrounds for different pixels are likely to be at different temperatures. Since the light received at the sensor focal-plane array is the combination of ground emission (as absorbed by the atmosphere) and atmospheric radiance, spatially varying ground temperature can produce spurious spectral features even if the ground emissivity is spectrally flat, as the ground emission and atmospheric radiance combine in different proportions.

One of many procedures for reducing the effects of ground emissivity and temperature variations makes use of a statistical analysis of the backgrounds in a scene; i.e., one constructs a spectral covariance matrix from the pixels, extracts principal components from the covariance matrix, and solves - in a least-squares fit - for the principal components of the background along with the chemicals of interest. ${ }^{15,16}$ It is superficially described here only to provide a little motivation for the incorporation of clutter effects into APRS.

Not all the effects of background clutter can be removed by this procedure. The residual clutter will depend strongly on the statistics of clutter in the background, how many principal components are included in the least-squares fit, the accuracy of those principal components (limited by sensor noise), the analyst's knowledge of the scene (e.g., of areas where the background should be nearly identical over all pixels), and random flukes (e.g., a car parked right under the plume).

\subsection{A clutter model}

A set of 129 material spectra from the Nonconventional Exploitation Factors Data System (NEFDS), ${ }^{14}$ comprising soils, asphalts, cements, paints, concretes, tars, bricks, cinderblocks, clays, sands, woods, gravels, and stones, was selected as representing stuff that might be found around plumes of interest.

These 129 material spectra could be distributed among pixels in an infinite number of ways. The most benign distribution would be if each and every pixel had precisely the same mixture of the 129 spectra and was at the same temperature - then the covariance matrix would have a single significant principal component (actually, just the average background), which when solved for in the least-squares fit mentioned above, would completely remove the background. A much worse, and more realistic, distribution would have each pixel containing a different material. A still worse distribution would in addition have each pixel at a different temperature.

The clutter model in APRS in effect assumes that 129 off-plume pixels are available, that each of these pixels has a single material as background, that no two pixels have the same background, and that each pixel is at the same temperature. For each of the reflectivity spectra, APRS calculates a quantity $\left(\tau^{\prime}\right)$ that is in fact produced by the spectral variability of the emissivity or reflectivity but, with an assumption of constant emissivity or reflectivity, would be interpreted as a chemical absorption. This quantity is calculated for each spectral bin or at each laser line. Principal components are extracted from the $129 \tau^{\prime}$ spectra with each material weighted identically. To do this, the covariance matrix is constructed as follows:

$$
\Lambda_{c}(i, j)=\frac{1}{129} \sum_{n=1}^{129}\left[\tau_{n}^{\prime}\left(\nu_{i}\right)-\left\langle\tau_{n}^{\prime}\left(\nu_{i}\right)\right\rangle\right] \times\left[\tau_{n}^{\prime}\left(\nu_{j}\right)-\left\langle\tau_{n}^{\prime}\left(\nu_{j}\right)\right\rangle\right]
$$

where

$$
\left\langle\tau_{n}^{\prime}\left(\nu_{i}\right)\right\rangle=\frac{1}{129} \sum_{n=1}^{129} \tau_{n}^{\prime}\left(\nu_{i}\right)
$$

and $\nu_{i}$ represents the center frequency of the $i^{t h}$ frequency bin or the frequency of the $i^{t h}$ laser line for an active system. Note that atmospheric transmission and instrument response are not included in this covariance matrix.

The covariance matrix is diagonalized via singular value decomposition, and the principal components are extracted. (Not all principal components are used.) The average of all the spectra - the average background - is also used; it has to be, because the average has been subtracted from each spectrum to form the covariance matrix in Eq. 38 .

A subset of the principal components (PCs) is included with the spectra of the chemicals of interest in a least-squares solution for the chemical concentration-pathlength (CL) products; the least-squares solution is a fit of the PC and chemical 
spectra to the fictitious chemical absorption signal ( $\tau^{\prime}$, described above) that would be measured for each reflectivity spectrum. (Each chemical absorption signal has the mean $\tau^{\prime}$ spectrum subtracted.) The maximum size of the subset of PCs used depends on the kind of sensor considered. Several considerations go into choosing this maximum subset size:

- An imaging sensor has much more background information than a non-imaging sensor;

- An active sensor must spend time off plume to acquire background spectral information, hence less time on plume;

- As more principal components are included in a least-squares solution for chemical CL, the NECL increases;

- As more principal components are included in a least-squares solution for chemical CL, the residual clutter effects decrease.

The first consideration can be accounted for by limiting the number of principal components available for a non-imaging system. Since a non-imaging system can have some spatial information available, a reasonable limit seemed to be ten principal components plus the average background. The second consideration can be accounted for by reducing on-plume time (for an active sensor).

Because of the third and fourth considerations above, a sensor will generally have an optimal number of principal components to be included. The location of this optimum will depend on how the chemical NECLs are combined with the residual effects of clutter.

The residual effects of clutter are evaluated by cycling through each of the 129 background spectra (in effect, cycling through the 129 pixels) and for each, determining the fictitious chemical CL that would be generated by the least-squares fit to that background. The background reflectivity is used for active systems and for reflected sunlight in passive systems, and the background emissivity is used for thermal radiation in passive systems. The fictitious chemical CL is evaluated with some number of principal components included in the least-squares fit. The 129 background spectra will generate 129 fictitious CLs. The residual effects of clutter are characterized by a clutter-equivalent CL (CECL), taken to be the standard deviation of those 129 fictitious CLs.

Finally, the chemical NECLs are combined with the CECLs to generate system-equivalent CLs (SECL) by root-sumsquaring the NECL and CECL:

$$
S E C L=\sqrt{N E C L^{2}+C E C L^{2}} .
$$

It is this SECL that has a minimum as a function of the number of principal components included in the least-squares fit; the position of that minimum identifies the optimal number of principal components (at least for the specific clutter model in APRS).

\subsection{Deficiencies}

Many of the assumptions that go into this model are somewhat arbitrary: the selection of the backgrounds, their statistical distribution, the requirements for off-plume time in an active system, the limit on the size of the off-plume spectrallyvariable area, and the maximum practical number of principal components for a non-imaging passive system are examples.

In addition, the clutter that arises from variations in background temperature - hence in the relative contributions of ground emissivity and atmospheric radiance - is not included.

During the benchmarking activities pursued in the DOE trade studies work, it became clear that APRS is definitely not perfect, as would be expected from a model intended to be generic enough to conduct trade studies. Nevertheless, it was eventually judged quite adequate (although difficult to use, partly because of the large number of input parameters that can be varied) for the extensive trade studies conducted.

\section{ACKNOWLEDGMENTS}

The author is happy to acknowledge many useful discussions with Asher Blum, Paul Kuzmenko, and Chuck Stevens of LLNL, and Mark Schmitt, Bernard Foy, and George Busch of LANL. 
This work was performed under the auspices of the US Department of Energy by the University of California Lawrence Livermore NationalLaboratory under contract W-7405-ENG-48.

\section{REFERENCES}

1. S. A. Clough, F. X. Kneizys, G. P. Anderson, E. P. Shettle, J. H. Chetwynd, and L. W. Abreu, "FASCOD3: Spectral Simulation," in IRS '88: Current Problems in Atmospheric Radiation - Proceedings of the 1988 International Radiation Symposium, ed., J. Lenoble and J.-F. Geleyn, Deepak Publishing, Hampton, VA, 1989.

2. G. P. Anderson, J. Wang, M. L. Hoke, F. X. Kneizys, J. H. Chetwynd, L. S. Rothman, L. M. Kimball, R. A. McClatchey, E. P. Shettle, S. A. Clough, W. O. Gallery, L. W. Abreu, and J. E. A. Selby, "History of one family of atmospheric radiative transfer codes," Proc. SPIE 2309, 170-183, 1994.

3. L. S. Rothman, R. R. Gamache, R. H. Tipping, C. P. Rinsland, M. A. H. Smith, D. C. Benner, V. M. Devi, J.M. Flaud, C. Camy-Peyret, A. Perrin, A. Goldman, S. T. Massie, L. R. Brown, and R. A. Toth, "The HITRAN Molecular Database: Editions of 1991 and 1992,” J. Quant. Spectrosc. Radiat. Transfer 48, 469-507, 1992.

4. http://cfa-www.harvard.edu/HITRAN/

5. F. D. Kahn, “The Signal: Noise ratio of a suggested spectral analyzer,” Ap. J. 129, 518-521, 1959.

6. P. L. Hanst, S. T. Hanst, and G. M. Williams, Infrared Spectra for Quantitative Analysis of Gases, Infrared Analysis, Inc., 1995, and http://www.infrared-analysis.com/

7. S. W. Sharpe, R. L. Sams, T. J. Johnson, P. M. Chu, G. C. Roderick, and F. R. Guenther, "Creation of $0.1 \mathrm{~cm}^{-1}$ resolution, quantitative, infrared spectral libraries for gas samples," Proc. SPIE 4577, 12-24, 2002, and http://nwir.pnl.gov

8. R. R. Beland, "Propagation through atmospheric optical turbulence," in The Infrared and Electro-Optical Systems Handbook, Vol. 2: Atmospheric Propagation of Radiation, F. G. Smith, Ed., ERIM and SPIE, 1993.

9. P. B. Ulrich, , "Hufnagel-Valley profiles for specified values of the coherence length and isoplanatic patch angle," Tech Note WJSA/MA/TN-88-013, W. J. Schafer Associates Inc., 1988.

10. R. L. Fante, "Electromagnetic beam propagation in turbulent media," Proc. IEEE 63, 1669-1692, 1975.

11. M. Schmitt, B. McVey, B. Cooke, and G. Busch, "Comprehensive system model for $\mathrm{CO}_{2}$ DIAL," Proc. SPIE 2702, 95-103, 1996.

12. J. W. Goodman, "Statistical properties of laser speckle patterns," in Laser Speckle and Related Phenomena, J. C. Dainty, ed., Springer-Verlag, 9-75, 1984.

13. R. E. Warren, "Detection and discrimination using multiple-wavelength differential absorption lidar," Applied Optics 24, 3541-3545, 1985.

14. For more information about NEFDS, contact Eric Stenberg, stenberge @ nima.mil.

15. A. Hayden, R. Noll, "Remote trace gas quantification using thermal IR spectroscopy and digital filtering based on principal components of background scene clutter,” Proc. SPIE 3071, 158-168, 1997.

16. A. Hayden, E. Niple, B. Boyce, "Determination of trace-gas amounts in plumes by the use of orthogonal digital filtering of thermal emission spectra," Applied Optics 35, 2802-2809, 1996. 